\title{
STABILITY OF SYMMETRIC VORTEX FLOW OVER SLENDER BODIES AND POSSIBILITY OF CONTROL BY LOCAL GAS HEATING
}

\author{
V. I. Shalaev and I. V. Shalaev \\ Moscow Institute of Physics and Technology (State University) \\ Zhukovsky 140182, Russia
}

\begin{abstract}
Stability properties of vortex structure over slender bodies at high angles of attack and instability mechanism are the subjects of many experimental and computational studies, most of which are observed in $[1$, $2]$. However, the reasons and mechanism of spontaneous symmetric flow breaking are not established up to now. In the first part of the present paper, a criterion for asymmetry origin for slender conical bodies is obtained using slender body theory [3] and catastrophe theory [4]. The theoretical results are verified by comparison with experimental data [58]; they helped to explain most of experimental observations and numerical simulations. In the second part, based on the obtained criterion and numerical solutions of boundary layer equations evaluations are made to estimate an effectiveness of global flow structure control method using local volumetric or surface gas heating. Qualitative confirmation of these estimations was done in experiments $[7,8]$ with gas heating by plasma discharge.
\end{abstract}

\section{STABILITY ANALYSIS}

A flow over a slender pointed body of self-similar cross-section shape $r$ $=F(\theta) a(X)$ where $a(0)=0 ; X$ is scaled with the body length $l ; r$ and $\theta$ are the polar radius and angle of the cylindrical coordinates $(X, r, \theta)$ scaled with maximal transversal size $\delta l$ and maximal relative width $\delta$, respectively, is considered.

It is assumed that the body is symmetric with respect to the plane $Z=0$ where $(X, Y, Z)$ are the Cartesian coordinates; $Y$ and $Z$ are scaled with $\delta l$. The freestream velocity is $U_{\infty}$; the vertical freestream velocity component is 


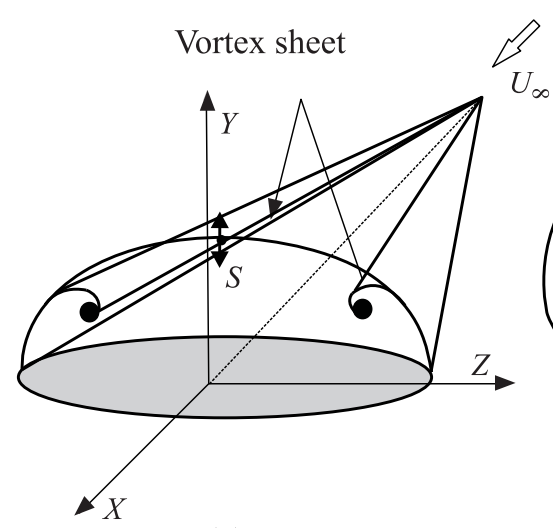

(a)

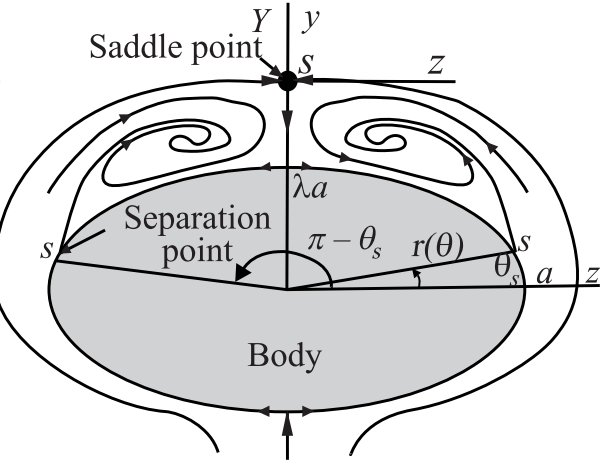

(b)

Figure 1 Basic flow scheme: (a) basic view; and (b) back view

$v_{\infty}^{*} \approx \alpha^{*} U_{\infty}$ where $\alpha^{*}$ is the angle of attack (Fig. $1 b$ ). It is assumed that the following relations are fulfilled:

$$
\delta=\frac{a_{0}^{*}}{l_{0}^{*}} \ll 1 ; \quad \delta \operatorname{Re}=\frac{U_{\infty} l_{0}^{*} \delta}{v_{\infty}} \gg 1 ; \quad \alpha=\frac{\alpha^{*}}{\delta}=O(1)
$$

where Re is the Reynolds number. At these conditions, the stability properties of the symmetric separated flow over slender body are determined by inviscid equations; it is verified by the analysis of the available literature $[1,2]$. In this approximation, the two separation angles, $\theta_{s}$ and $\pi-\theta_{s}$, are the parameters which can be determined from boundary layer calculations or experiments.

It is shown by majority of experiments and calculations that the symmetric vortex structure is not stable with respect to very small flow imperfections. However, theoretical studies [1] show that the linear approximation for perturbations leads to disagreement with experimental data. To derive the nonlinear model for perturbed flow, the asymptotic expansions of the flow parameters are used:

$$
\begin{gathered}
u^{*}=U_{\infty}\left[1+\delta^{2} \ln \delta u_{0}(X)+\delta^{2} u(X, Y, Z)+\cdots\right] \\
v^{*}=U_{\infty} \delta v(X, Y, Z)+\cdots ; \quad w^{*}=U_{\infty} \delta w(X, Y, Z)+\cdots ; \\
p^{*}-p_{\infty}=\rho_{\infty} U_{\infty}^{2} \delta^{2} p(X, Y, Z)+\cdots ; \quad \rho^{*}=\rho_{\infty}\left[1+\delta^{2} \rho(X, Y, Z)+\cdots\right] .
\end{gathered}
$$

Here, $u^{*}, v^{*}$, and $w^{*}$ are the components of the velocity vector in Cartesian coordinates; $p^{*}$ and $\rho^{*}$ are the pressure and density; and $p_{\infty}$ and $\rho_{\infty}$ are their freestream values. The perturbed flow in cross-section planes is described by the two-dimensional unsteady incompressible Euler equations: 


$$
\left.\begin{array}{rl}
\frac{\partial v}{\partial X}+v \frac{\partial v}{\partial Y}+w \frac{\partial v}{\partial Z}+\frac{\partial p}{\partial Y} & =0 \\
\frac{\partial w}{\partial X}+v \frac{\partial w}{\partial Y}+w \frac{\partial w}{\partial Z}+\frac{\partial p}{\partial Z} & =0 ; \\
\frac{\partial v}{\partial Y}+\frac{\partial w}{\partial z} & =0 ; \\
{\left[v-w B \frac{d F}{d Z}\right]_{Y=B F}} & =F \frac{d B}{d X}
\end{array}\right\}
$$

where streamwise coordinate $X$ is similar to time. Each solution of Eqs. (1) generates a streamline field that obeys the system of the following ordinary differential equations (ODE):

$$
\begin{gathered}
\frac{d Z}{d X}=w(X, Y, Z), \quad \frac{d Y}{d X}=v(X, Y, Z) \\
X=X_{0} ; \quad Y=Y_{0} ; \quad Z=Z_{0}
\end{gathered}
$$

Equation (3) specify the initial conditions for each streamline at the plane $X=X_{0}$.

Due to one-to-one correspondence between systems (1) and (2)-(3), one can study the stability of system (2)-(3) instead of Eqs. (1). Thus, the stability problem is reduced to the analysis of dynamic system stability utilizing the catastrophe theory [4]. The properties of singular points where $v=w=0$ determine qualitative behavior and stability of the dynamic system (2)-(3). The transition to asymmetric flow corresponds to the change of a singular point type.

Exact mathematical results exist only for autonomous dynamic systems [4]; in the considered problem, it corresponds to conical flows. In this case, $B=X$, the cone surface is described by equation $\eta=F(z), 2 \delta$ is the maximum cone apex angle, and the flow properties depend on conical variables:

$$
x=\ln X ; \quad \eta=\frac{Y}{X} ; \quad z=\frac{Z}{X} .
$$

The solution of Euler equation can be found in the class of discontinuous potential functions, so that the following relations are valid:

$$
\begin{aligned}
w(\eta, z) & =\frac{\partial \Phi}{\partial z} ; & v(\eta, z) & =\frac{\partial \Phi}{\partial \eta} \\
\frac{\partial^{2} \Phi}{\partial z^{2}}+\frac{\partial^{2} \Phi}{\partial \eta^{2}} & =0 ; & {\left[\frac{\partial \Phi}{\partial \eta}-\eta-\left(\frac{\partial \Phi}{\partial z}-z\right) \frac{d F}{d z}\right]_{\eta=F(x)} } & =F
\end{aligned}
$$


Here, $\Phi(\eta, z)$ is the flow potential. The ODE Eqs. (2)-(3) in conical variables (4) have the following form:

$$
\frac{d \eta}{d x}=v-\eta ; \quad \frac{d z}{d x}=w-z
$$

Qualitative behavior of the autonomous dynamic system (5) is determined by the properties of singular points where $v=w=0$. Bifurcation of solution arises at appearance of a doubly degenerated critical point where the Hessian of system (5) is equal to zero [4], that is

$$
\left(\frac{\partial v}{\partial \eta}-1\right)\left(\frac{\partial w}{\partial z}-1\right)-\frac{\partial v}{\partial z} \frac{\partial w}{\partial \eta}=0 .
$$

Let consider the flow scheme shown in Fig. $1 b$ that corresponds to separated flow at sufficiently large angles of attack. In this vortex flow, the following singular points are present: two vortex centers, the half-saddles separation points at $\theta=\theta_{s}$ and $\pi-\theta_{s}$, two attachment points at $\theta=0$ and $\pi$, and the saddle point $S$ which is formed at the angle of attack exceeding a critical value [1, 2]. The first six points are Morse saddles in terms of [4] do not change their types at transition to asymmetry, only the saddle point $S$ can change its type. A set of these saddle points forms a streamline that for symmetrical flow is determined by the equations $\eta=\eta_{S}, z=0$. In the vicinity of point $S$, taking into account the potential and symmetry conditions, the flow velocity components are decomposed to the Taylor series:

$$
\begin{array}{rlrl}
v & =\eta_{S}-a y+b y^{3}-2 b z^{2} y ; & w & =a z+b z^{3}-3 b y^{2} z ; \\
y & =\eta-\eta_{S} ; & a\left(\alpha, \theta_{s}\right) & =-\frac{\partial v\left(\eta_{S}, 0\right)}{\partial \eta}=\frac{\partial w\left(\eta_{S}, 0\right)}{\partial z} ; \\
b\left(\alpha, \theta_{s}\right) & =\frac{1}{6} \frac{\partial^{3} v\left(\eta_{S}, 0\right)}{\partial \eta^{3}}=\frac{1}{6} \frac{\partial^{3} w\left(\eta_{S}, 0\right)}{\partial z^{3}} ; \frac{\partial v\left(\eta_{S}, 0\right)}{\partial z}=\frac{\partial w\left(\eta_{S}, 0\right)}{\partial \eta}=0 .
\end{array}
$$

In the vicinity of the point $S$, Eqs. (6) are reduced to the form:

$$
\left.\begin{array}{rlrl}
\frac{d z}{d x} & =-c_{1} z+b z^{3}-3 b y^{2} z ; & \frac{d y}{d x} & =-c y+b y^{3}-3 b z^{2} y ; \\
\left(\alpha, \theta_{s}\right) & =1-a\left(\alpha, \theta_{s}\right) ; \quad c\left(\alpha, \theta_{s}\right)=1+a\left(\alpha, \theta_{s}\right) .
\end{array}\right\}
$$

For Eqs. (7), the symmetric flow stability criterion (6) is reduced to the following relation:

$$
c\left(\alpha, \theta_{s}\right) c_{1}\left(\alpha, \theta_{s}\right)=0 .
$$

Thus, at a bifurcation point, one of coefficients is equal to zero, $c_{1}=0$ or $c=0$, which is equivalent to $a=1$ or -1 . 


\section{STABILITY CRITERION CALCULATION AND VERIFICATION}

To simplify calculations, the symmetric vortex flows over slender cones are simulated using the model [3] and conformal mapping of the body cross-section on the unit circle. In this model, the concentrated vortices and the vortex sheets are mimicked by the point vortices and line cuts connecting the separation points with vortices. The Kutta-Zhukovsky condition at the separation points and the equilibrium condition for the vortex system lead to three nonlinear algebraic equations:

$$
P_{i}\left(\mathbf{x}, \theta_{s}, \alpha\right)=0, \quad i=1,2,3,
$$

where $\mathbf{x}=\left(x_{1}, x_{2}, x_{3}\right)$, for vortex intensity $x_{1}$ and vortex coordinates $x_{2}$ and $x_{3}$. The separation angle $\theta_{s}$ and angle of attack $\alpha$ are the parameters. The
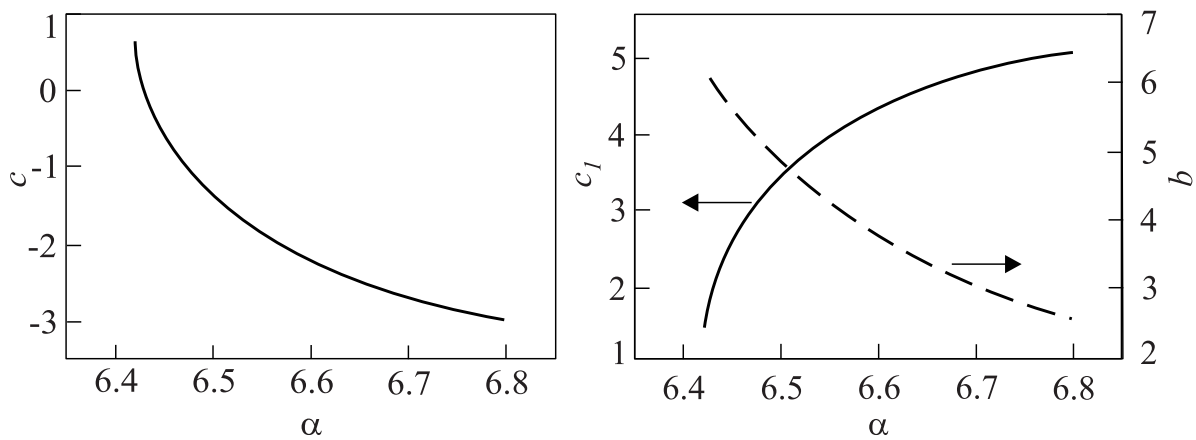

(a)
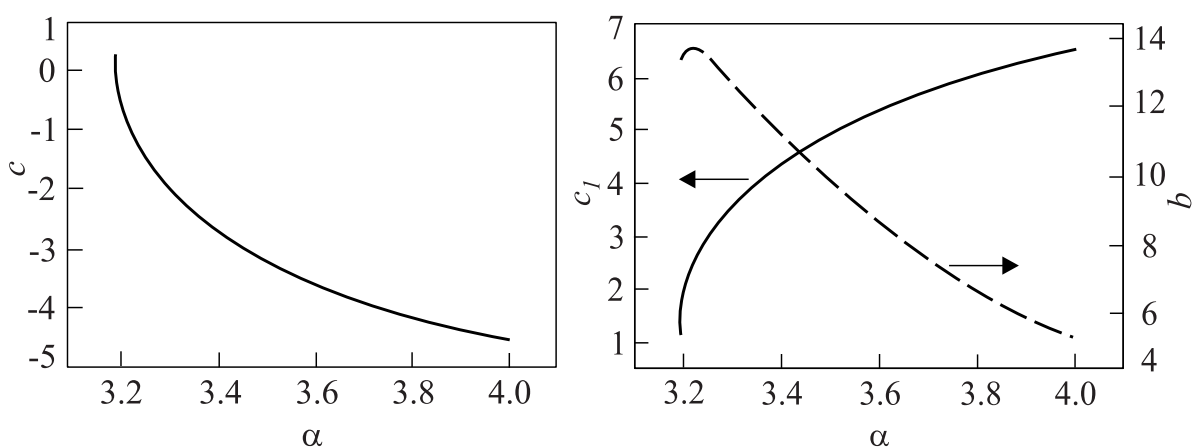

(b)

Figure 2 Coefficients of Eqs. (7) for round cone $\lambda=1$ : (a) $\theta_{s} \approx 0^{\circ}$; and (b) $\theta_{s} \approx 40^{\circ}$ 

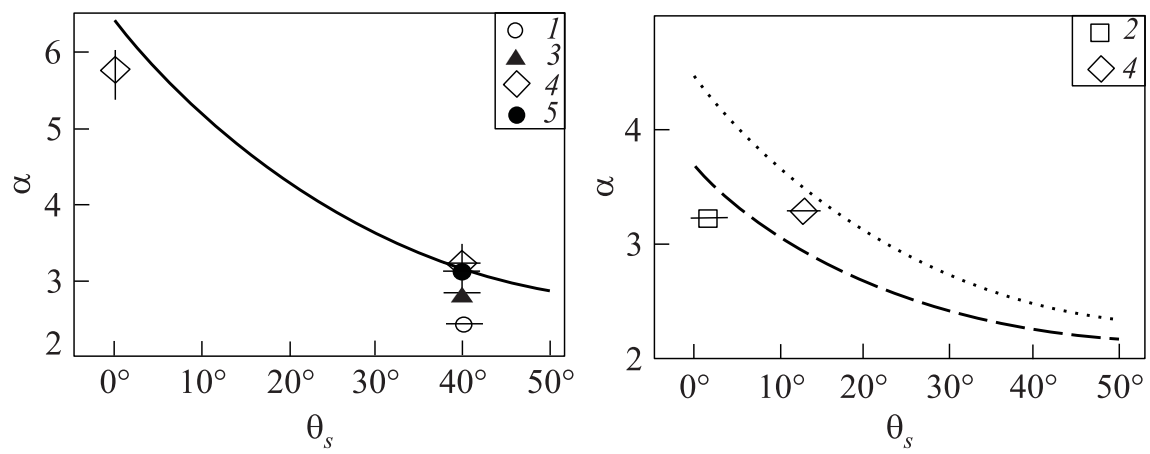

Figure 3 The stability criteria $c\left(\alpha, \theta_{s}\right)=0$ for round $(\lambda=1)(a)$ and elliptic (dashed curve $-\lambda=0.3$, and dotted curve $-\lambda=0.5)(b)$ cones: $1-\delta=10^{\circ}[6] ; 2-10^{\circ}[7$, $8] ; 3-5^{\circ}[6] ; 4-5^{\circ}[7,8] ;$ and $5-\delta=3^{\circ}[6]$

calculations were conducted for elliptic cones with minor axis $\lambda X, 0<\lambda \leq 1$; the values $\lambda=1$ and 0 correspond to a round cone and a delta wing, respectively.

Typical behavior of coefficients $c(\alpha), c_{1}(\alpha)$, and $b(\alpha)$ for round cone $(\lambda=1)$ is presented in Fig. 2: $\theta_{s} \approx 0^{\circ}$ (Fig. $2 a$ ) corresponds to laminar boundary layer separation; $\theta_{s} \approx 40^{\circ}$ (Fig. $2 b$ ) corresponds to turbulent boundary layer separation. Parametric studies showed that the coefficients $c_{1}\left(\alpha, \theta_{s}\right)$ and $b\left(\alpha, \theta_{s}\right)$ are always positive, and $c\left(\alpha, \theta_{s}\right)$ change its sign for each separation angle when angle of attack is varied. From these properties, it follows that bifurcation criterion can be written as $c\left(\alpha, \theta_{s}\right)=0$.

In Figs. $3 a$ and $3 b$, the calculated curves $c\left(\alpha, \theta_{s}\right)=0$ for round and elliptic cones are shown by curves. Symbols correspond to experimental data of asymmetric state origin [6-8]; vertical and horizontal segments show the measurement accuracy of normalized angle of attack and separation angle. The data $[7,8]$ for round cone were obtained in the wind tunnel T-324 of Institute of Theoretical and Applied Mechanics of the Siberian Branch of the Russian Academy of Sciences with low level of freestream perturbations and high level of cone surface smoothness; the turbulent regime was created using turbulators.

The utilized asymptotic approach is appropriate at the limit $\delta \rightarrow 0$. The data for round cones of different apex angles in Fig. $3 a$ [6] demonstrate the convergence of the theory and experiments with the cone apex angle diminishing for the turbulent boundary layer separation $\left(\theta_{s} \approx 40^{\circ}\right)$. For the thinner cone $\left(\delta=3^{\circ}\right)$, the experimental results are absent; in this case, the extrapolation of $[6]$ is used and this value almost matches the calculation result. The calculations agree well with the experiments $[7,8]$ (4, see Fig. $3 a$ ), as for the turbulent $\left(\theta_{s} \approx 40^{\circ}\right)$ and laminar $\left(\theta_{s} \approx 0^{\circ}\right)$ separation. In spite of the rough flow model 
and large apex angle $\left(\delta=10^{\circ}\right)$ the stability criteria in Fig. $3 b$ for elliptic cones with $\lambda=0.3$ and 0.5 conforms satisfactorily to experiments [6].

Note that the bifurcation diagram of asymmetric solutions of the model [3] differs from the presented results since the saddle point $S$ arises in the flow at larger angles of attack $[1,2,6]$.

The catastrophe theory [4] gives only the criterion of asymmetry origin but cannot describe the transition process. However, some qualitative results of the beginning of this process can be obtained by the analysis of local streamline Eqs. (7). The transition to asymmetric state is associated with the type change of the critical point $S$, when there is a change of sign of the coefficient $c\left(\alpha, \theta_{s}\right)$, and with the global flow instability at $c\left(\alpha, \theta_{s}\right)<0$. Calculations for elliptic cones show that $b>0, c_{1}>3 c$. Thus, critical points of Eqs. (7) are: the symmetric point $y=z=0(S))$ and the following points:

$$
\begin{aligned}
& c<0: \quad y=0, \quad z=\sqrt{\frac{c_{1}}{b}}\left(S_{1}\right) ; \quad y=0, \quad z=-\sqrt{\frac{c_{1}}{b}}\left(S_{2}\right) ; \\
& c>0: \quad S_{1}, S_{2} ; z=0, \quad y=\sqrt{\frac{c}{b}}\left(S_{3}\right) ; \quad z=0, \quad y=-\sqrt{\frac{c}{b}}\left(S_{4}\right) .
\end{aligned}
$$

At $c<0$, three nondegenerated critical points $\left(S, S_{1}\right.$, and $\left.S_{2}\right)$ exist at the axis $y=0 ; S$ is the unstable saddle. At $c=0$, critical points $S, S_{3}$, and $S_{4}$ coincide, i. e., $S$ is triply degenerated in the $y$-direction and has the saddle-node type. At $c>0$, there are five nondegenerated critical points: $S ; S_{1}$ and $S_{2}$ at the axis $y=0$; and $S_{3}$ and $S_{4}$ at the axis $z=0 ; S$ is the unstable node.

For qualitative analysis of transition to asymmetry, let consider the second equation of Eqs. (7) in the plane $z=0$, where it is reduced to the simplest form of the Ginzburg-Landau equation [9]:

$$
\frac{d y}{d x}=-c y+b y^{3} \text {. }
$$

At $c \neq 0$ and $b>0$, Eq. (8) has the following solution:

$$
h(X)=y^{2}=h_{c}\left[1-B_{0}\left(\frac{X}{X_{0}}\right)^{2 c}\right]^{-1},
$$

where

$$
B_{0}=\frac{h_{0}-h_{c}}{h_{0}} ; \quad h_{c}=\frac{c}{b} .
$$

The condition $h\left(X_{0}\right)=h_{0}$ specifies an initial streamline perturbation in the plane $z=0$. 
At $c>0$, the solution of Eq. (8) is stable in the linear approximation $(b=0)$ :

$$
y=y_{0}\left(\frac{X}{X_{0}}\right)^{-c} \rightarrow 0, \quad X \rightarrow \infty .
$$

For $h_{0}<h_{c}$, the coefficient $B_{0}<0$ and the nonlinear solution (9) is also stable: $h(X) \rightarrow 0$ at $X \rightarrow \infty$, i. e., streamlines remain in attraction region of the stable node $S$. For $h_{0}>h_{c}$, the coefficient $B_{0}>0$ and $h(X) \rightarrow \infty$ at $X \rightarrow X_{c}$ where

$$
X_{c}=X_{0} B_{0}^{-1 /(2 c)} ; \quad Y_{c}= \pm X_{0} \sqrt{\frac{c}{b}}= \pm X_{0} \sqrt{h_{c}} .
$$

Here, $X_{c}$ is the length of perturbation development; it is a linear function of $X_{0}$ and nonlinear function of $h_{0}$. Thus, at $h_{0}>h_{c}$ and $c>0$, streamlines leave the attraction region of the stable node $S$ at the finite distance from the cone apex. The value $Y_{c}$ characterizes the threshold level of stationary perturbation at the plane $X=X_{0}$ that initiates instability. This threshold and $X_{c}$ are decreased when the initial data plane is shifted to the cone apex: $Y_{c} \rightarrow 0, X_{c} \rightarrow 0$ at $X_{0} \rightarrow 0$. So, at $h_{0}>h_{c}$ and $c>0$, solution (9) describes the subcritical bifurcation under the influence of finite perturbations.

If $c<0$ the solution (9) is unstable in linear and nonlinear approximations since the coefficient $B_{0}>0$ and $h(X) \rightarrow \infty$ at $X \rightarrow X_{c}$.

For $c=0$, the solution of Eq. (9) has the following form:

$$
h=h_{0}\left[1-2 b h_{0} \ln \left(\frac{X}{X_{0}}\right)\right]^{-1} ; \quad X_{c}=X_{0} \exp \left(\frac{1}{2 b h_{0}}\right) .
$$

This is also nonlinear unstable solution where the transition region depends on initial perturbation exponentially.

Although the study of flows being influenced by external disturbances is beyond the frame of this paper, it can be stated that for the mentioned more complicated case, the qualitative behavior of flow is similar to that of obtained above for the symmetric flow. The presented results show that the asymmetry origin mechanism is essentially nonlinear. Due to the nonlinearity, it is impossible to extract the perturbations from general equations and to study them separately. There is a finite length where perturbations are amplified to infinity, and this distance is diminished if the initial data plane is shifted to the body apex. The subcritical bifurcation is possible at angles of attack lower than the critical value, if the initial perturbation exceeds a threshold, which value decreases with shifting of the initial data plane to the body apex.

The instability mechanism has the properties of both convective and absolute instability where the coordinate $X$ is treated as time. However, this terminology relates to linear theory of hydrodynamic stability and cannot fully describe the considered nonlinear process. This instability can be characterized more exactly 
as global or structural instability. The presented qualitative analysis and the asymmetry origin criterion allow to explain most of the effects found in the experiments and numerical simulations. The developed approach allows also to analyze unsteady perturbations [2].

\section{SEPARATION CONTROL USING LOCAL VOLUMETRIC AND SURFACE GAS HEATING}

The second problem considered in this paper is the analysis of asymmetry control possibility using the local gas heating by plasma discharge. The idea of utilization of such approach to global flow structure control has been proposed in $[1,2,7$, $8,10]$. The theoretical verification of this idea is presented hereafter.

For analysis of the problem, the local self-similar approximation for threedimensional turbulent boundary layer on a round slender cone surface is utilized. The plasma discharge effect is modeled by heat release in the boundary layer. The effect of gas ionization is neglected since the ionization coefficient is of the order of $10^{-5}$. The flow scheme and the coordinate system are shown in Fig. 4. The flow scheme shows that heat release impacts the boundary layer as an obstacle.

To simplify the boundary layer equations, the Dorodnitsyn-Stewartson transformation is used along the normal coordinate $y^{*}$ :

$$
d \eta=\sqrt{\frac{\operatorname{Re}}{x}} \rho d \frac{y^{*}}{l}, \quad \operatorname{Re}=\frac{\rho_{\infty} u_{\infty} l}{\mu_{\infty}} .
$$

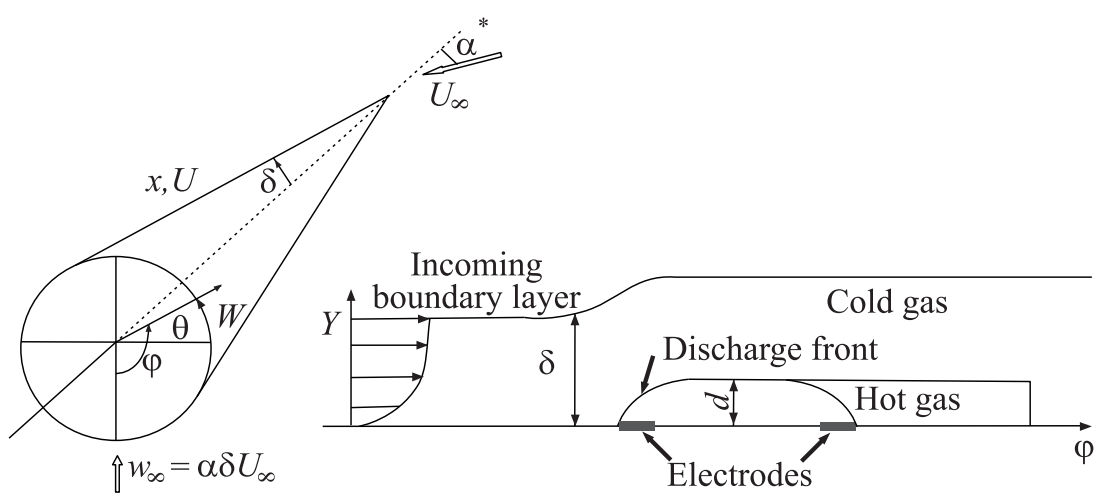

(a)

(b)

Figure 4 Coordinate system $(a)$ and flow scheme $(b)$ for the boundary layer 
The self-similar boundary-layer equations and boundary conditions are expressed as

$$
\left.\begin{array}{c}
V \frac{\partial U}{\partial \eta}+w_{e} W \frac{\partial U}{\partial \varphi}=\frac{\partial}{\partial \eta}\left(m \frac{\partial U}{\partial \eta}\right) ; \\
V \frac{\partial h}{\partial \eta}+w_{e} W \frac{\partial h}{\partial \varphi}-Q(x, y, \varphi)=\frac{\partial}{\partial \eta} k \frac{\partial h}{\partial \eta} ; \\
V \frac{\partial W}{\partial \eta}+w_{e} W \frac{\partial W}{\partial \varphi}+U W+\frac{\partial w_{e}}{\partial \varphi} W^{2}-h\left(1+\frac{\partial w_{e}}{\partial \varphi}\right)=\frac{\partial}{\partial \eta}\left(m \frac{\partial W}{\partial \eta}\right) ; \\
\rho h=1 ; \quad V=-\left(\frac{3}{2} f+\frac{\partial w_{e}}{\partial \phi} g+w_{e} \frac{\partial g}{\partial \phi}\right) ; \\
U=\frac{\partial f}{\partial y} ; \quad W=\frac{\partial g}{\partial y} ; \quad h=\frac{\partial e}{\partial y} ; \\
y=\infty: U=W=h=1 ; \quad y=0: U=W=\frac{\partial h}{\partial y}=0 .
\end{array}\right\}
$$

Here, the angle $\varphi=\theta+0.5 \pi, f(x, y, \varphi)$ and $g(x, y, \varphi)$ are the stream functions; axial speed $U$, density $\rho$, and enthalpy $h$ are scaled to their freestream values denoted by index $\infty$. The transverse speed $W$ is normalized by $\delta U_{\infty} \omega_{e}$ and the circumferential velocity at the upper boundary-layer edge, $w_{e}(\varphi)$ is scaled to $\delta U_{\infty}$. For turbulent flows, the normalized heat conductivity $k$ and viscosity $m$ are determined using the Cebeci-Smith model as

$$
\begin{gathered}
m=\rho \mu(1+\varepsilon) ; \quad k=\frac{\rho \mu}{\operatorname{Pr}}\left(1+\frac{\operatorname{Pr}}{\operatorname{Pr}_{t}} \varepsilon\right) ; \\
0 \leq y \leq y_{w}: \quad \varepsilon=\varepsilon_{1}\left(\frac{\partial U}{\partial y}\right)^{2} ; \\
y>y_{w}: \quad \varepsilon=0.0168 \sqrt{\operatorname{Re} x} D_{1} \frac{\delta_{u}}{h \mu} ; \\
\varepsilon_{1}=0.16 D_{1} \frac{\sqrt{\operatorname{Re} x}}{\mu}\left(\frac{e D}{h}\right)^{2} ; \quad \delta_{u}=\int_{0}^{y_{e}}(1-U) d y \\
D=1-\exp (-d) ; \quad d=(\operatorname{Re} x)^{1 / 4} \frac{e}{26 h_{w}} \sqrt{\frac{1}{\mu_{w}} \frac{\partial U}{\partial y}} \\
D_{1}=\left[1+\left(\frac{e}{e_{e}}\right)^{6}\right] ; \quad \mu=h^{3 / 2} \frac{1+h_{S}}{h+h_{S}} ; \quad h_{S}=\frac{110.4}{T_{\infty}}
\end{gathered}
$$

Here, the subscript $w$ corresponds to the parameters on the wall. 
In the left-hand side of the energy equations (10), the heat source term is represented in the form

$$
Q=\frac{Q^{*} x l}{h_{\infty} U_{\infty}}=Q_{0} y^{2} \exp \left[-\frac{\left(y-y_{c}(\varphi)\right)^{2}}{\sigma}\right] ; \quad y_{c}=2 y_{0} \sqrt{\left|\left(\varphi-\varphi_{1}\right)\left(\varphi_{2}-\varphi\right)\right|} .
$$

Here, $Q^{*}$ is the dimensional source intensity; $Q_{0}$ is the maximum of dimensionless heat release intensity; $\sigma$ characterizes the discharge width; $y_{c}(\varphi)$ is the centerline of the discharge that is approximated by the parabola; $y_{0}$ is the maximum distance from the discharge centerline to the wall, the angles $\varphi_{1}$ and $\varphi_{2}$ determine the electrode locations. The total power released in the boundary layer is determined as

$$
P=\iiint_{V_{h}} \rho^{*} Q^{*} a^{*}(x) d x^{*} d \phi d y^{*}=\frac{\beta \rho_{\infty} h_{\infty} U_{\infty} l^{2}}{\sqrt{\operatorname{Re}}} \int_{x_{1}}^{x_{2}} \sqrt{x} d x \int_{\varphi_{1}}^{\varphi_{2}} d \varphi \int_{0}^{\infty} Q d y .
$$

If $Q_{0}$ is the function of the coordinate $x$ and/or the flow is turbulent, then the boundary layer is not self-similar. However, one can use Eqs. (10) for the first-order estimations of flow characteristics. The calculations of the boundary layer characteristics were conducted for the inviscid flow approximated by $w_{e}=2 \alpha \sin \varphi$. Some calculation results are presented below for the following parameters: $\alpha=3.15 ; \delta=5^{\circ} ; l=1 \mathrm{~m} ; T_{\infty}=288.15 \mathrm{~K}, U_{\infty}=10 \mathrm{~m} / \mathrm{s} ; \sigma=1$; $y_{0}=1$; the center between electrodes is located at $\phi_{0}=0.5\left(\phi_{1}+\phi_{2}\right)=1.714 \mathrm{rad}$ $\left(98.25^{\circ}\right) ; \phi_{1}=\phi_{0}-3 \Delta \phi ; \phi_{2}=\phi_{0}+3 \Delta \phi$ where $\Delta \phi=0.0314159$ is the integration step of the finite-difference approximation.

In Fig. 5, the temperature (Fig. 5a) and circumferential velocity (Fig. 5b) profiles across the boundary layer are shown as the functions of $\eta$ for $Q_{0}=200$

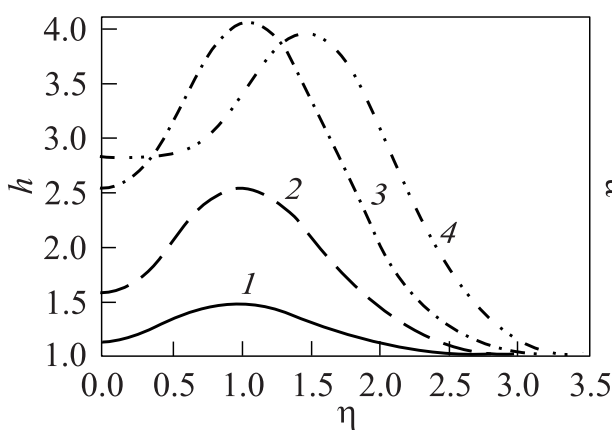

(a)

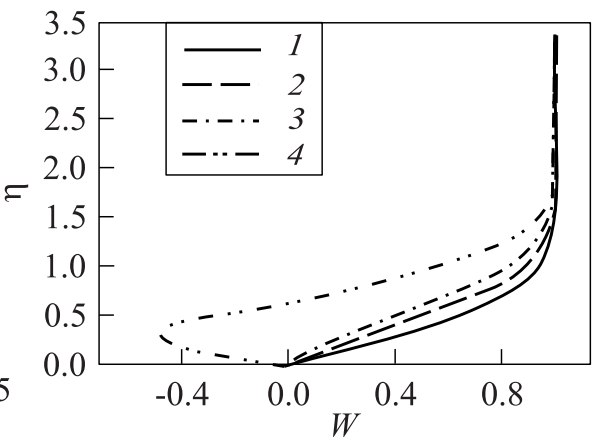

(b)

Figure 5 Profiles of temperature $(a)$ и and circumferential velocity $(b)$ across the boundary layer near heat release region for $Q_{0}=200: 1-\phi=1.64 ; 2-1.71 ; 3-$ $1.81 ;$ and $4-\phi=1.87$ 


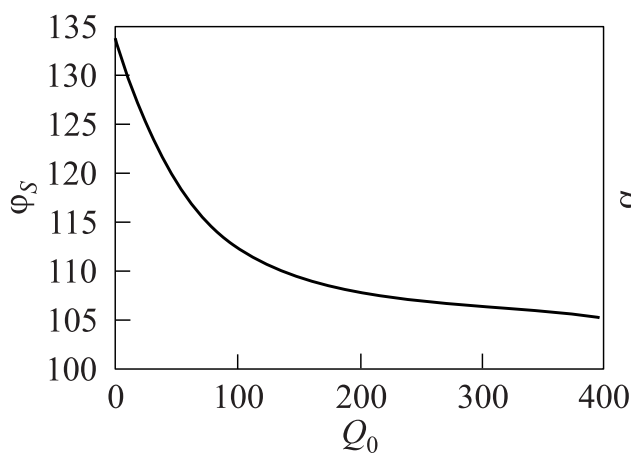

(a)

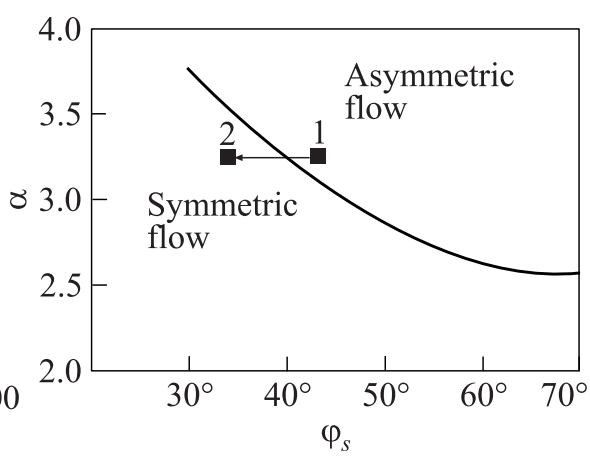

(b)

Figure 6 Discharge effect on the separation angle $(a)$ and flow state $(b)$

and for different polar angles $\varphi$. The temperature profiles are similar to the source heat intensity distribution across the boundary layer. The temperature reaches its maximum value near the rear electrode, $\varphi=\varphi_{2}=1.809$. Behind the heat source region, the temperature maximum decreases and moves toward the upper boundary-layer edge due to the heat diffusion. The station $\varphi=1.87$ is located just after the separation point.

Figure $6 a$ demonstrates the plasma discharge effect on the separation point. As the heat-source intensity increases from 0 to 400 , the separation angle, $\varphi_{s}$, decreases from $133^{\circ}$ to about $105^{\circ}$. It is seen that the plasma heating is more effective in the range $Q_{0}<100$, where the slope $d \varphi_{s} / d Q_{0}$ is relatively large. Figure $6 b$ illustrates the feasibility of the vortex structure control using a local boundary-layer heating. The solid line in this figure represents the calculated in section 1 boundary between symmetric and asymmetric flow states. Due to the heat release, the flow configuration changes from the initial asymmetric state $\left(\varphi_{s} \approx 133^{\circ}\right.$, symbol 1$)$ to the symmetric state with $\theta_{s} \approx 120^{\circ}$ (symbol 2 ). This requires a nondimensional heat source intensity $Q_{0} \approx 30$. Using Eqs. (11), it is possible to estimate that the corresponding total power is approximately equal to $480 \mathrm{~W}$. This example indicates that the method is feasible for practical applications of the global flow structure control [10].

Another method to control the flow separation consisting of the body surface local heating is also analyzed in this paper for the case of slender round cone. The boundary layer characteristics were calculated using Eqs. (11) with $Q=0$, and the boundary condition for enthalpy was changed to $h(0, \varphi)=h_{w}$ where $h_{w}$ is the normalized wall temperature. The flow scheme for this case is shown in Fig. 7. The whole cone surface had the temperature $T_{w}=T_{\infty}\left(h_{w}=1\right)$ except for the narrow strip $\Delta \varphi$ that had the temperature $T_{w}=T_{\text {strip }}>T_{\infty}$. The boundary layer calculations were conducted for the cone of the length $1 \mathrm{~m}$ 


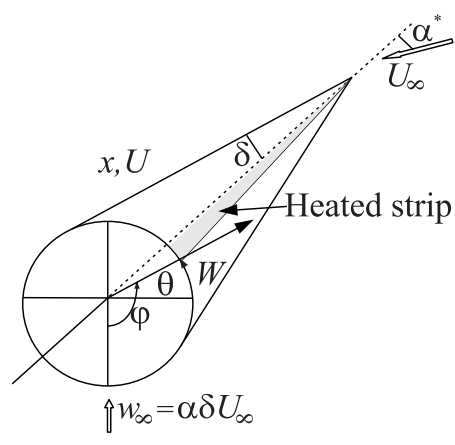

(a)

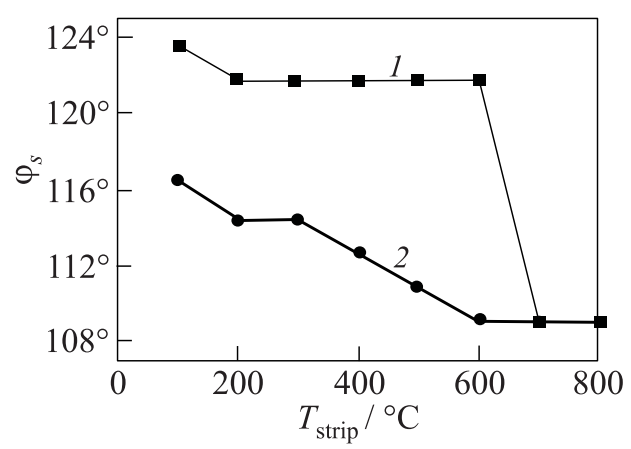

(b)

Figure 7 Flow scheme for cone with heated strip $(a)$ and effect of strip temperature on separation angle $(b): 1-\alpha=3$; and $2-\alpha=4$

with apex angle $2 \delta=10^{\circ}$ at the freestream velocity $20 \mathrm{~m} / \mathrm{s}$, and normalized angles of attack $\alpha=2-4$ The freestream temperature was $T_{\infty}=0{ }^{\circ} \mathrm{C}$, the strip temperature varied in the range of $T_{n}=100-800{ }^{\circ} \mathrm{C}$, the strip width was $\Delta \varphi=12^{\circ}$, and its center was located at $\varphi=110^{\circ}$.

The separation angles $\varphi_{s}$ as functions of strip temperature $T_{\text {strip }}$ are presented in Fig. $7 b$ for $\alpha=3$ and 4 . For $\alpha=2$, the separation angle does not depend on strip temperature. At $\alpha=3$, the separation angle $\varphi_{s}$ decreases from $133^{\circ}$ to $122^{\circ}$ with strip temperature increasing to $200{ }^{\circ} \mathrm{C}$. The separation angle is constant up to $T_{\text {strip }}=600{ }^{\circ} \mathrm{C}$, then it varies to $\varphi_{1 s} \approx 109^{\circ}$ as strip temperature increases to $700{ }^{\circ} \mathrm{C}$, and stabilizes at this value at subsequent strip temperature growth. For $\alpha=4$, the separation angle decreases monotonically to $\varphi_{1 \mathrm{~s}} \approx 109^{\circ}$, if the strip is heated from 100 to $600{ }^{\circ} \mathrm{C}$, and remains constant at further temperature growth.

The results presented in this section show that the local surface heating can be used for control of separation location; however, this method is less effective than the volumetric gas heating.

\section{CONCLUDING REMARKS}

In this paper, the criterion for transition to asymmetric state of the vortex flow over round and elliptic pointed cones is obtained using slender body theory and catastrophe theory. The calculations are verified by comparison with experimental data. The qualitative analysis of transition properties based on symmetric flow streamlines behavior study is presented. The analysis shows that the transi- 
tion is a nonlinear process and it is characterized by a finite development length and threshold level of perturbation, which are diminished if the initial data plane is shifted to the cone apex.

The presented studies of separation angle control in a turbulent boundary layer using the local volumetric and surface gas heating show that both methods can be used for flow global structure control but the first one is more effective since the separation angle displacement is larger in the first case at approximately same gas temperature level.

\section{REFERENCES}

1. Shalaev, V., A. Fedorov, N. Malmuth, V. Zharov, and I. Shalaev. 2003. Plasma control of forebody nose symmetry breaking. AIAA Paper No. 2003-0034.

2. Shalaev, V., A. Fedorov, N. Malmuth, and I. Shalaev. 2004. Plasma control of forebody nose symmetry breaking. AIAA Paper No. 2004-0842.

3. Dyer, D.E., S.P. Fiddes, and J.H.B. Smith. 1982. Asymmetric vortex formation from cone at incidence - a simple inviscid model. Aeronautical Quarterly 33(6):293-312.

4. Gilmore, R. 1981. Catastrophe theory for scientists and engineers. New York.

5. Keener, E. R., and G. T. Chapman. 1977. Similarity in vortex asymmetries over slender bodies and wings. AIAA J. 15(9):1370-72.

6. Lowson, M. V., and A. J. C. Ponton. 1992. Symmetric breaking in vortex flows on conical bodies. AIAA J. 30(6):1576-83.

7. Fomin, V. M., A. A. Maslov, A. A. Sidorenko, et al. 2004. Control of vortex flow over bodies of revolution by electric discharge. Dokl. Russ. Acad. Sci. 396(5):1-4. [In Russian.]

8. Maslov, A., B. Zanin, A. Sidorenko, et al. 2004. Plasma control of separated flow asymmetry on a cone at high angle of attack. AIAA Paper No. 2004-0843.

9. Landau, L. D., and E. M. Lifshits. 1986. Theoretical physics, V. VI: Hydrodynamics. Moscow. [In Russian.]

10. Shalaev, V., A. Fedorov, N. Malmuth, et al. 2004. Surface plasma discharge for controlling forebody vortex asymmetry. U.S. Patent 6796532. 